\title{
Economic impact of antifungal treatment in IFD by Candida species: anidulafungin administration as a cost-effective alternative for critical care patients
}

\author{
F Cattel, ${ }^{1}$ F Ancona, ${ }^{2}$ M Chiumente, ${ }^{3}$ A Miliacca ${ }_{1}^{4}$ I Motta, ${ }^{5}$ F G De Rosa ${ }^{6}$
}

${ }^{1}$ S.C Farmacia, A.O. Città della salute e della scienza di Torino, Turin, Italy

${ }^{2}$ Partner

PricewaterhouseCoopers Advisory SpA, Turin, Italy ${ }^{3}$ Post Graduate School of Hospital Pharmacy, Università degli Studi di Torino, Turin, Italy

${ }^{4}$ Manager

PricewaterhouseCoopers Advisory SpA, Rome, Italy ${ }^{5}$ Post Graduate School of Infectious Diseases, Università degli Studi di Torino, Turin, Italy

${ }^{6}$ Department of Medical Sciences, University of Turin, Turin, Italy

\section{Correspondence to} Dr Francesco Cattel, AOU S. Giovanni Battista Hospital, Cap 10126 C.so Bramante, 88/90 Torino, Turin, Italy: Fcattel@gmail.com

Received 8 October 2013 Revised 15 March 2014 Accepted 22 May 2014 Published Online First 5 June 2014
CrossMark

\author{
To cite: Cattel F, Ancona $F$, \\ Chiumente $\mathrm{M}$, et al. Eur J \\ Hosp Pharm 2015;22:
} 12-17.

\section{ABSTRACT}

Objectives To evaluate the potential savings resulting from the substitution of caspofungin and liposomal amphotericin B with anidulafungin in intensive care units (ICUs), where appropriate, for the treatment of invasive fungal disease.

Methods We conducted a retrospective analysis on patients admitted to two ICUs at S. Giovanni Battista hospital in Turin (IT). Included patients were admitted during the year 2010 for $\geq 3$ days. We evaluated the substitutability index of caspofungin and liposomal amphotericin B with anidulafungin. According to the index, we created an economical model in order to project the potential savings for the entire hospital. Results A total of 179 medical records were included in the analysis. We evaluated 488 doses of antifungals administered, including: 174 anidulafungin, 209 caspofungin and 105 liposomal amphotericin B. Anidulafungin was found to be a possible substitute for a total of 166 doses of caspofungin and 43 doses of liposomal amphotericin B. The substitutability index was $79.4 \%$ and $40.9 \%$, respectively, for caspofungin and liposomal amphotericin B. The projected savings would be $€ 37300$ for all of the departments examined. If this antifungal drug had not been included in the hospital formulary, and thus not administered in the two ICUs, the hospital would have spent $€ 42747$ more. According to our model the differential cost is equal to 37300 $+42747=€ 80047$.

Conclusions The savings we obtained is proportional to our substitutability index based on a sample of patients admitted to our ICUs. We assume that highly specialised hospitals may obtain an index higher than the one we obtained. Therefore, greater savings proportional to the number of patients treated are expected.

\section{INTRODUCTION}

Invasive fungal disease (IFD) may be severe in critically ill patients, where Candida species infections may range ${ }^{12}$ from mucocutaneous disease to bloodstream infections. ${ }^{3}$ Candidemia represents 10-20\% of cases of invasive candidiasis (IC) and has a high mortality rate $(38-75 \%)$ with consistent variations in epidemiology. ${ }^{4-7}$ The risk factors for IFD by Candida species include the use of central venous catheters, mechanical ventilation, total parenteral nutrition, surgery (particularly abdominal), prolonged stays in the intensive care unit (ICU), immunocompromised status and steroid administration. ${ }^{8-11}$

The incidence of IFD caused by Candida species in critically ill patients may be grossly underestimated due to the wide use of precocious empirical antifungal treatment guided by several scores, such as the Leon score, ${ }^{12}$ or because of death before diagnosis of IFD. ${ }^{13}$ The treatment of IFD may be expensive and several authors have published economic evaluations with variable costs depending on the comorbidities and aetiology. ${ }^{5} 14$ Together with infectious disease specialists and microbiologists, hospital pharmacists currently have a key role in the cost management related to treatment, including the monitoring and appropriateness of prescriptions. The antimicrobial stewardship programmes now include an antifungal stewardship, ensuring appropriate treatment according to the available guidelines. ${ }^{15-17}$ According to the Infectious Diseases Society of America (IDSA) guidelines, the first line of treatment for critically ill patients with IFD by Candida species is based on echinocandins, such as caspofungin, anidulafungin, micafungin or liposomal amphotericin B, particularly in cases that require biofilm-active agents. ${ }^{18}{ }^{19}$ However, as part of a broader optimisation of purchasing management and rationalisation of inventory and logistics, several health administrators have decided not to include all three echinocandins in the available formularies, due to more extensive indications for caspofungin or micafungin as compared with anidulafungin.

In this study, we retrospectively evaluated the 'index of substitutability' of caspofungin and liposomal amphotericin B with anidulafungin in two ICUs, if appropriate and then with equal effectiveness according to IDSA guidelines ${ }^{19}$ and official therapeutic indications, ${ }^{20}$ to evaluate the potential economic advantage of anidulafungin administration.

The echinocandin micafungin has not been included in our study because until 2010 this drug was not present in the hospital formulary.

\section{MATERIALS AND METHODS}

This retrospective study was carried out in two critical care wards at San Giovanni Battista Hospital - 'Città della Salute e della Scienza di Torino', a reference hospital located in northern Italy with solid organ and bone marrow transplant programmes. All echinocandins are available for clinical use; anidulafungin has been available since 2009 .

In the first phase of the study, the medical records of critically ill patients, including immunocompromised and solid organ transplant recipients, who were admitted for $\geq 3$ days during 2010 were reviewed and the use of antifungals was detailed. The following data were collected by a pharmacist and an infectious diseases specialist: identification code of the patient, date of admission, age and 
gender, total days of hospital and ICU stay, reason for ICU admission, outcome, diagnostic tests for IFD, and type, duration, and dosage of antifungal treatment (fluconazole, caspofungin, anidulafungin and liposomal amphotericin B).

Micafungin had not been included in the hospital formulary until then.

The second phase of the study was represented by an economic analysis of the potential use of anidulafungin, when caspofungin or liposomal amphotericin B was administered. In particular, the cost of anidulafungin was compared with the two other drugs in the empirical treatment of candidemia or IC. Exclusion criteria for the change to anidulafungin were represented by known or suspected IFD by Aspergillus species and neutropenia due to haematological disease.

\section{Analysis of the economic impact of anidulafungin use on the hospital budget}

The economic analysis was based on several factors, including: the cost of daily administration of anidulafungin, caspofungin and liposomal amphotericin B; the number of cases where anidulafungin could be used (according to Agenzia Italiana del Farmaco directions) in patients with empirical or targeted treatment of IC or candidemia; and the index of substitution of anidulafungin.

\section{Cost of daily administration of anidulafungin}

The purchase price per pack given by the hospital management software was considered. The cost of the package was based on daily administration according to the average quantity of product typically administered. The average amount administered was obtained by calculating the average of the quantity dispensed on the first administration with the quantities of subsequent administrations dispensed throughout the duration of the therapy.

Cost of the daily dose of caspofungin and liposomal amphotericin $B$, where anidulafungin represents a potential substitute

As described above, we calculated the daily administration cost of caspofungin and liposomal amphotericin $\mathrm{B}$, the drug candidates for substitution. Hence, the concept of the differential cost of administration was defined as the difference between the cost of anidulafungin and the cost of caspofungin or liposomal amphotericin B.

Number of cases where anidulafungin may be used as an alternative to the other antifungals

In Italy, anidulafungin use is officially indicated for the 'treatment of IC in non-neutropenic patients. ${ }^{20}$

To assess the potential substitutability of caspofungin and amphotericin B with anidulafungin maintaining equal effectiveness, for each patient included in the analysis, the infectious disease specialists of the antimicrobial stewardship evaluated several clinical parameters extracted from medical records, according to IDSA guidelines ${ }^{19}$ and official therapeutic indications. ${ }^{20}$

The 'index of substitutability' of anidulafungin with caspofungin or liposomal amphotericin B was calculated by detecting the number of delivered doses of caspofungin and liposomal amphotericin B and the number of cases in which it would have been equally appropriate to administer anidulafungin.

After identifying the number of caspofungin doses provided (c) and the number of cases where it would be equally appropriate to administer anidulafungin $\left(a_{c}\right)$, the index of substitutability' of anidulafungin compared with caspofungin was defined as the ratio $a_{d} / c$. The 'index of substitutability' of anidulafungin to liposomal amphotericin $B(f)$ was defined as the ratio $a_{f} / f$, where $a_{f}$ represents the number of cases where anidulafungin served as an appropriate alternative to liposomal amphotericin B.

These two indices of substitutability vary according to departmental and hospital settings, due to the different patient distributions (eg, proportion of post-transplant recipients) and the distinct microbial ecologies.

\section{Method of calculation}

The cost of the daily administration of anidulafungin was determined according to the official dosage, with a loading dose of $200 \mathrm{mg}$ for the 1 st day and $100 \mathrm{mg}$ daily thereafter to complete the 14-day treatment. Therefore, the cost of daily administration is equal to the cost per milligram for the amount administered, according to the following formula:

$$
\mathrm{C}_{\mathrm{A}}=\mathrm{Cmg}_{\mathrm{A}} \bullet \mathrm{QM}_{\mathrm{A}}
$$

Definitions and basic values: $\mathrm{C}_{\mathrm{A}}=$ Daily administration cost of anidulafungin, $\mathrm{Cmg}_{\mathrm{A}}=\mathrm{Cost}$ per milligram of anidulafungin (€3.22), $\mathrm{QM}_{\mathrm{A}}=$ Average daily amount $(107.14 \mathrm{mg})$.

The cost of the daily administration of drugs potentially substitutable with anidulafungin was similarly determined. The following formulas were used to identify the cost of daily administration of caspofungin $\left(\mathrm{C}_{\mathrm{C}}\right)$ and the cost of daily administration of liposomal amphotericin $\mathrm{B}\left(\mathrm{C}_{\mathrm{F}}\right)$, respectively. The average daily dose $(\mathrm{QM})$ for both drugs was obtained from the medical records.

The cost of the daily administration of caspofungin was calculated by multiplying the cost per milligram by the amount administered, according to the following formula:

$$
\mathrm{C}_{\mathrm{C}}=\mathrm{Cmg}_{\mathrm{C}} \bullet \mathrm{QM}_{\mathrm{C}}
$$

Definitions and basic values: $\mathrm{C}_{\mathrm{C}}=$ Daily administration cost of caspofungin, $\mathrm{Cmg}_{\mathrm{C}}=$ Cost per milligram of caspofungin $(€ 7.66)$, $\mathrm{QM}_{\mathrm{C}}=$ Average daily amount $(52.25 \mathrm{mg})$.

To determine the cost of the daily administration of liposomal amphotericin $\mathrm{B}$, the following formula was applied:

$$
\mathrm{C}_{\mathrm{F}}=\mathrm{Cmg}_{\mathrm{F}} \bullet \mathrm{QM}_{\mathrm{F}}
$$

Definitions and basic values: $\mathrm{C}_{\mathrm{F}}=$ Daily administration cost of liposomal amphotericin $\mathrm{B}, \mathrm{Cmg}_{\mathrm{F}}=$ Cost per milligram of liposomal amphotericin B $(€ 2.67), \quad \mathrm{QM}_{\mathrm{F}}=$ Average daily amount (238.45 mg).

The differential daily-dose costs were calculated according to the following formulas:

$$
\begin{aligned}
\Delta \mathrm{C}_{\mathrm{C}} \mathrm{C}_{\mathrm{A}} & =\mathrm{C}_{\mathrm{C}}-\mathrm{C}_{\mathrm{A}} \\
\Delta \mathrm{C}_{\mathrm{F}} \mathrm{C}_{\mathrm{A}} & =\mathrm{C}_{\mathrm{F}}-\mathrm{C}_{\mathrm{A}}
\end{aligned}
$$

Definitions: $\Delta \mathrm{C}_{\mathrm{C}} \mathrm{C}_{\mathrm{A}}=$ Daily differential cost (savings) of anidulafungin compared with caspofungin, $\Delta \mathrm{C}_{\mathrm{F}} \mathrm{C}_{\mathrm{A}}=$ Daily differential cost (savings) of anidulafungin compared with liposomal amphotericin $\mathrm{B}, \mathrm{C}_{\mathrm{C}}=$ Daily administration cost of caspofungin, $\mathrm{C}_{\mathrm{A}}=$ Daily administration cost of anidulafungin, $\mathrm{C}_{\mathrm{F}}=$ Daily administration cost of liposomal amphotericin B.

The medical records examined included most, but not all, of the total consumption of antifungals administered in the two ICUs considered.

To identify the total substitutable doses of caspofungin and liposomal amphotericin B in the two wards considered, in addition to the 'index of substitutability' as previously defined, we 
Table 1 Patient characteristics

\begin{tabular}{llllll}
\hline $\begin{array}{l}\text { Number of } \\
\text { patients }(\mathbf{n})\end{array}$ & Men $(\mathbf{n})$ & Women $(\mathbf{n})$ & $\begin{array}{l}\text { Age (mean } \pm \text { SD) } \\
\text { (years) }\end{array}$ & $\begin{array}{l}\text { Overall days of } \\
\text { hospitalisation (mean } \pm \text { SD) }\end{array}$ & $\begin{array}{l}\text { Days of hospitalisation } \\
\text { in ICU (mean } \pm S D)\end{array}$ \\
\hline 179 & 114 & 65 & $64.31 \pm 14.63$ & $52.62 \pm 4.75$ & $16.86 \pm 1.74$ \\
\hline
\end{tabular}

ICU, intensive care unit.

projected our data to the total consumption of antifungals within the two ICUs.

To calculate the savings resulting from the replacement of caspofungin with anidulafungin in the two wards observed, the following formula was adopted:

$$
\text { Saving }=\mathrm{IS}_{\mathrm{AC}} \times \mathrm{ND}_{\mathrm{C}} \times \Delta \mathrm{C}_{\mathrm{C}} \mathrm{C}_{\mathrm{A}}
$$

Definitions and basic values: $\mathrm{IS}_{\mathrm{AC}}=$ Index of substitutability of anidulafungin compared with caspofungin, $\mathrm{ND}_{\mathrm{C}}=\mathrm{Number}$ of total doses of caspofungin consumed in the considered wards as measured by the stock movements (231), $\Delta \mathrm{C}_{\mathrm{c}} \mathrm{C}_{\mathrm{A}}=$ Daily differential cost (savings) of anidulafungin compared with caspofungin.

The following formula was used to calculate the savings resulting from the switch from amphotericin B to anidulafungin:

$$
\text { Saving }=\mathrm{IS}_{\mathrm{AF}} \times \mathrm{ND}_{\mathrm{F}} \times \Delta \mathrm{C}_{\mathrm{F}} \mathrm{C}_{\mathrm{A}}
$$

Definitions and basic values: IS $_{\mathrm{AF}}=$ Index of substitutability of anidulafungin compared with liposomal amphotericin B, $\mathrm{ND}_{\mathrm{F}}=$ Number of total doses of liposomal amphotericin B consumed in the considered wards as measured by the stock movements (228.14), $\Delta \mathrm{C}_{\mathrm{F}} \mathrm{C}_{\mathrm{A}}=$ Daily differential cost (savings) of anidulafungin compared with liposomal amphotericin $\mathrm{B}$.

The sum of the values obtained to this point was used to determine the overall saving if anidulafungin were administered in place of caspofungin and liposomal amphotericin B.

We then measured the total differential cost between two extreme scenarios: the administration of anidulafungin in all cases where, according to our definition, anidulafungin was deemed appropriate versus no administration of anidulafungin (representing a lack of drug availability in the hospital formulary).

The first element to be determined was the number of potential doses of anidulafungin (K). This was equal to the sum of the number of doses of anidulafungin actually delivered and the number of replaceable administrations, respectively, of caspofungin and liposomal amphotericin B.

$\mathrm{K}=$ number of doses of anidulafungin + number of replaceable caspofungin doses + number of replaceable liposomal amphotericin $\mathrm{B}$ doses. $\mathrm{K}$ represents the potential amount of administrable daily doses of anidulafungin.

The total cost, if anidulafungin were dispensed in $100 \%$ of the cases, would be equal to: $\mathrm{K} \times \mathrm{C}_{\mathrm{A}}$.

On the contrary, in the absence of anidulafungin administration, it is assumed that all doses would have been distributed between caspofungin and liposomal amphotericin B. Therefore, the doses of caspofungin and liposomal amphotericin B were determined according to the following formulas:

$$
\begin{aligned}
\mathrm{D}_{\mathrm{C}} & =\mathrm{K} \times\left[\mathrm{DS}_{\mathrm{C}} /\left(\mathrm{DS}_{\mathrm{C}}+\mathrm{DS}_{\mathrm{F}}\right)\right] \\
\mathrm{D}_{\mathrm{F}} & =\mathrm{K} \times\left[\mathrm{DS}_{\mathrm{F}} /\left(\mathrm{DS}_{\mathrm{C}}+\mathrm{DS}_{\mathrm{F}}\right)\right]
\end{aligned}
$$

Definitions and basic values: $\mathrm{Dc}=$ Number of doses of caspofungin administered in absence of anidulafungin, $D_{F}=$ Number of doses of amphotericin B administered in absence of anidulafungin, $\mathrm{K}=$ Number of potential administrations of anidulafungin, $\mathrm{DS}_{\mathrm{C}}=$ Number of doses of caspofungin replaceable with anidulafungin (183.47), $\mathrm{DS}_{\mathrm{F}}=$ Number of doses of liposomal amphotericin B replaceable with anidulafungin (93.43).

In the scenario involving no administration of anidulafungin, the total cost would be equal to the sum of the daily dose values of caspofungin and liposomal amphotericin B. The total cost in the absence of anidulafungin administration would be:

$$
\mathrm{D}_{\mathrm{C}} \times \mathrm{C}_{\mathrm{C}}+\mathrm{D}_{\mathrm{F}} \times \mathrm{C}_{\mathrm{F}}
$$

The overall differential cost (savings) was obtained from the resulting difference between the total cost in the absence of anidulafungin administration and the total cost of anidulafungin administered in $100 \%$ of the cases.

\section{RESULTS}

A total of 179 medical and/or surgical patients were included in the analysis from data collected by a pharmacist and an infectious diseases specialist (table 1).

The costs of daily administration of anidulafungin $\left(\mathrm{C}_{\mathrm{A}}\right)$, caspofungin $\left(\mathrm{C}_{\mathrm{C}}\right)$ and liposomal amphotericin $\mathrm{B}\left(\mathrm{C}_{\mathrm{F}}\right)$ were: $€ 345.12$, $€ 400.08$ and $€ 636.42$, respectively. The daily differential cost (savings) of anidulafungin compared with caspofungin, equal to $C_{C}-C_{A}$ (400.08-345.12), was $€ 54.96$, and the daily differential cost (savings) of anidulafungin compared with liposomal amphotericin $B$, equal to $C_{F}-C_{A}(636.42-345.12)$, was $€ 291.31$.

We evaluated 209 doses of caspofungin, 166 of which were deemed replaceable with anidulafungin (according to Agenzia Italiana del Farmaco indications). The substitutability index, according to the ratio $(166 / 209) \times 100 \%$, was $79.43 \%$.

We also determined that, of the 105 doses of liposomal amphotericin B assessed, 43 were replaceable with anidulafungin, resulting in a substitutability index of $40.95 \%$ (table 2 ).

\section{Calculation of the impact on the two ICUs' budget}

The substitutability indices have been projected to all consump-

\begin{tabular}{|c|c|c|c|}
\hline Drug & $\begin{array}{l}\text { Administered } \\
\text { doses }\end{array}$ & $\begin{array}{l}\text { Potential } \\
\text { substitutable } \\
\text { doses by } \\
\text { anidulafungin }\end{array}$ & $\begin{array}{l}\text { Substitutability } \\
\text { index }\end{array}$ \\
\hline Caspofungin & 209 & 166 & $79.43 \%$ \\
\hline Liposomal amphotericin B & 105 & 43 & $40.95 \%$ \\
\hline Anidulafungin & 174 & & \\
\hline Total cases observed & 488 & 209 & $\mathrm{n} / \mathrm{a}$ \\
\hline
\end{tabular}
tions of caspofungin and liposomal amphotericin B in the two ICUs, as measured by stock movements recorded by the hospital pharmacy.

Table 2 Doses and substitutability index 
Table 3 Total savings following use of anidulafungin as an alternative for other antifungal drugs

\begin{tabular}{|c|c|c|c|c|c|}
\hline Drug & $\begin{array}{l}\text { Total doses administered } \\
\text { detected by management software }\end{array}$ & Substitutability index & Substitutable doses & $\begin{array}{l}\text { Differential saving for } \\
\text { daily administration }\end{array}$ & Total saving \\
\hline Caspofungin & $231.00\left(\mathrm{ND}_{\mathrm{C}}\right)$ & $79.43 \%\left(I_{A C}\right)$ & 183.47 & $€ 54.96\left(\Delta C_{C} C_{A}\right)$ & $€ 10083.71$ \\
\hline Liposomal amphotericin B & $228.14\left(N D_{F}\right)$ & $40.95 \%\left(I S_{A F}\right)$ & 93.43 & $€ 291.31\left(\Delta C_{F} C_{A}\right)$ & $€ 27216.29$ \\
\hline TOTAL & & & & & $€ 37300.00$ \\
\hline
\end{tabular}

The total savings with anidulafungin administration instead of caspofungin in the two critical wards, based on differential costs equal to $€ 54.96$, is $€ 10083.71$.

The savings obtained from switching to anidulafungin from liposomal amphotericin $\mathrm{B}$, based on the relative differential cost equal to $€ 291.31$, is $€ 27216.29$.

Therefore, the total savings would be: $€ 10083.71+$ $€ 27216.29=€ 37300$ (table 3).

Moreover, we calculated the total and the differential costs in two hypothetical settings: first, if anidulafungin was not available and, second, if a complete substitution with anidulafungin were feasible.

The first element determined was the number of potential doses of anidulafungin (K).

$\mathrm{K}$ is the sum of the doses of anidulafungin actually provided (317.33), the exchangeable doses of caspofungin (183.47) and the exchangeable doses of liposomal amphotericin B (93.43), equalling 594.23.

In the case of anidulafungin absence, it is assumed that all doses would have been distributed between caspofungin and liposomal amphotericin B.

Therefore, in the absence of anidulafungin, the doses of administrable caspofungin or liposomal amphotericin B would have been 393.73 and 200.5, respectively.

As a result, in the first scenario, the total cost would be equal to $€ 205081(594.23 \times 345.12)$.

In the second scenario, the total cost of $€ 285128$ was obtained by applying the percentages, illustrated in table 4, which refer to the potential amount of daily doses (594.23): 393.74 doses of caspofungin and 200.50 doses of liposomal amphotericin B.

The overall savings, resulting from the two settings, totals $€ 80047$ (€285 128-€205 081).

If we subtract from overall savings obtained by the two extreme scenarios (€80.047), the savings arising from introduction of anidulafungin in our hospital ( $€ 37.300)$, according to the substitutability index calculated, we obtain a total of $€ 42$ 747, the savings achieved in 2010 due to the introduction of anidulafungin in the hospital formulary.

The savings increase proportionally with the number of anidulafungin doses administered as compared with the doses potentially administered, the amphotericin B and caspofungin substitutable doses (figure 1).

These results are based upon the purchase price incurred by the pilot hospital. In order to obtain more interesting results at a national level, we have repeated all the calculations starting from the reference prices supported by most of the hospitals in the country. Ecalta and Cancidas have the same price everywhere in Italy, corresponding to ex-factory price. For Ambisome, Molinette hospital pays $€ 1631.1$ for a $50 \mathrm{mg}$, 10 -dose package, with a $50 \%$ discount on the official price, while most hospitals pay $€ 1957.32$ (40\% discount). If we substituted the latter in the calculations we would obtain a total saving of $€ 108406$.

\section{DISCUSSION}

The incidence of IFDs is on the rise and treatment of IC or candidemia with echinocandins or liposomal amphotericin B may be costly, especially considering the empirical treatment in the intensive care setting. US and European guidelines recommend an echinocandin as the best available option for patients with candidemia and IC, ${ }^{21}$ and liposomal amphotericin B when an antibiofilm agent is needed. ${ }^{22}$ In critical care settings, there are a number of predictive rules that help in identifying patients at risk of developing candidemia, such as the Ostrozky, Leon, Paphitou, and other scores. ${ }^{23-25}$ Sensitivity and specificity range from approximately $41 \%$ to $81 \%$ and $61 \%$ to $86 \%$, respectively.

Our study was designed to assess the potential savings within the ICUs, if caspofungin or liposomal amphotericin B were substituted with anidulafungin to treat known or suspected IFD by Candida species, thus excluding mould disease caused by Aspergillus species. In our study, the savings achieved by the possible administration of anidulafungin, as measured by the 'index of substitutability', were greater when compared with liposomal amphotericin B than caspofungin.

Beyond the savings, there are special properties of anidulafungin in the critical care setting, such as the highest volume of distribution among the echinocandins. Anidulafungin was found to be non-inferior to fluconazole for the treatment of IC in a major randomised clinical trial, with a faster time to negative cultures. Anidulafungin was effective in an ICU non-neutropenic population of patients with candidemia or IC and with $\geq 1$ of the following: post abdominal surgery; age $\geq 65$ years; renal/ hepatic insufficiency; solid organ transplant; and/or solid tumour. $^{26}$ Moreover, anidulafungin was determined to be a cost-effective option by an economic analysis that investigated anidulafungin compared with fluconazole for the treatment of IC in an Australian hospital perspective. The study used an analytical decision model that was constructed to capture

Table 4 Total cost without anidulafungin

\begin{tabular}{|c|c|c|c|c|c|}
\hline Drug & Replaceable doses & Frequency & Spreading doses of anidulafungin & Cost per dose & Total cost \\
\hline Caspofungin & $183.47\left(D_{C}\right)$ & $66.26 \%$ & $393.73\left(D_{C}\right)$ & $€ 400.08\left(C_{C}\right)$ & $€ 157.523$ \\
\hline Liposomal amphotericin B & $93.43\left(\mathrm{DS}_{\mathrm{F}}\right)$ & $33.74 \%$ & $200.5\left(D_{F}\right)$ & $€ 636.42\left(C_{F}\right)$ & $€ 127.602$ \\
\hline Total & 276.9 & $100.00 \%$ & 594.23 & & $€ 285.125$ \\
\hline
\end{tabular}




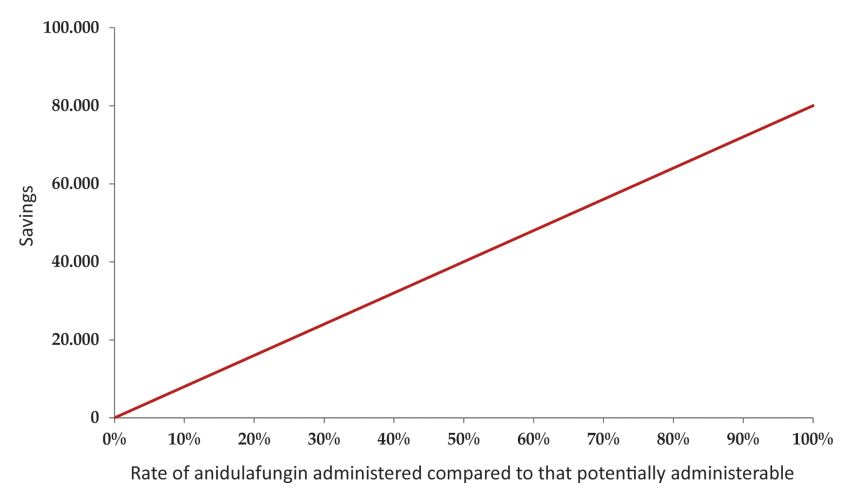

Figure 1 Trend of potential savings within the two intensive care units.

downstream consequences of using either agent for the treatment of IC, with the main outcomes set as treatment success and treatment failure. ${ }^{27}$

Generally, most analyses are still based on pharmacoeconomic modelling rather than direct analysis of trial data or real life clinical populations. The antifungal treatment basically includes administration for suspected or proven infection and the most important cost drivers in antifungal therapy are represented by early initiation of antifungal therapy, adjustment after availability of microbiological results, duration of therapy, therapeutic target, and the occurrence of severe complications, such as renal failure. $^{28}$

While caspofungin, the most extensively studied echinocandin, was previously determined to be the most cost-effective

\section{Key messages}

What is already known on this subject

- The treatment of invasive fungal disease may be expensive and several authors have published economic evaluations with variable costs depending on the comorbidities and aetiology.

- The new antifungal anidulafungin is indicated for treatment of invasive candidiasis and has a lower cost per day of treatment than other drugs with comparable indication.

- In scientific literature, for antifungals, there are several economic evaluations based on cost-efficacy rate but none was suited to our reality, with Italian costs and the peculiar organisation of our Italian hospitals.

What this study adds

- In this study, we retrospectively evaluated the 'index of substitutability' of caspofungin and liposomal amphotericin $B$ with anidulafungin in two intensive care units to evaluate the potential economic advantage of anidulafungin administration according to the official indications.

- The savings achieved by the possible administration of anidulafungin, as measured by the 'index of substitutability', were greater when compared with liposomal amphotericin B than with caspofungin.

- We developed an economic model able to quantify the savings resulting from use of anidulafungin, where appropriate, instead of more expensive drugs with broader indications; this model provides a useful tool for understanding the potential savings arising from the inclusion of anidulafungin in the hospital formulary. option for the treatment of IC and as an empirical therapy of suspected infections, our specific model found anidulafungin the most cost-effective antifungal option, especially compared with liposomal amphotericin B. There are inherent limitations to our single-centre study, including the retrospective nature and the large population of patients with solid organ transplant and haematopoietic stem cell transplant at our institution. Thus, the results of our study may not be generalisable to those conducted at other institutions.

The savings we obtained is proportional to our index of substitutability.

The potential savings cannot be extended based on the number of beds available in another hospital; such extrapolation would require the consideration of the risk of candida infections in the population of patients treated and the official costs specific of other settings. We assume that highly specialised hospitals may obtain an 'index of substitutability' higher than the one obtained from this study, and therefore, greater savings proportional to the number of patients treated.

Competing interests None.

Ethics approval

Provenance and peer review Not commissioned; externally peer reviewed.

\section{REFERENCES}

1 Pagano L, Caira M, Nosari A, et al. Fungal infections in recipients of hematopoietic stem cell transplants: results of the SEIFEM B-2004 study—Sorveglianza Epidemiologica Infezioni Fungine Nelle Emopatie Maligne. Clin Infect Dis 2007;45:1161-70.

2 Piazza 0, Boccia MC, lasiello A, et al. Candidemia in intensive care patients. Risk factors and mortality. Minerva anestesiologica 2004;70:63-9.

3 Tortorano AM, Dho G, Prigitano A, et al. Invasive fungal infections in the intensive care unit: a multicentre, prospective, observational study in Italy (2006-2008). Mycoses 2012;55:73-9.

4 Jarvis WR. Epidemiology of nosocomial fungal infections, with emphasis on Candida species. Clin Infect Dis 1995;20:1526-30.

5 Wey SB, Mori M, Pfaller MA, et al. Hospital-acquired candidemia. The attributable mortality and excess length of stay. Arch Intern Med 1988;148: 2642-5.

6 Falcone M, Russo A, De Rosa FG, et al. Clinical experience of anidulafungin for the treatment of patients with documented candidemia. J Chemother 2010;22:397-401.

7 Bassetti M, Taramasso L, Nicco E, et al. Epidemiology, species distribution, antifungal susceptibility and outcome of nosocomial candidemia in a tertiary care hospital in Italy. PLOS ONE 2011;6:e24198.

8 Martino R, Subira M, Rovira $M$, et al. Invasive fungal infections after allogeneic peripheral blood stem cell transplantation: incidence and risk factors in 395 patients. Br J Haematol 2002;116:475-82.

9 Jantunen $E$, Ruutu $P$, Niskanen $L$, et al. Incidence and risk factors for invasive fungal infections in allogeneic BMT recipients. Bone Marrow Transplant 1997; 19:801-8.

10 Kam LW, Lin JD. Management of systemic candidal infections in the intensive care unit. Am J Health Syst Pharm 2002;59:33-41.

11 Bedini A, Venturelli C, Mussini C, et al. Epidemiology of candidaemia and antifungal susceptibility patterns in an Italian tertiary-care hospital. Clin Microbiol Infect 2006;12:75-80.

12 Leon C, Ruiz-Santana S, Saavedra P, et al. Usefulness of the "Candida score" for discriminating between Candida colonization and invasive candidiasis in non-neutropenic critically ill patients: a prospective multicenter study. Crit Care Med 2009;37:1624-33.

13 Kami M, Machida U, Okuzumi K, et al. Effect of fluconazole prophylaxis on fungal blood cultures: an autopsy-based study involving 720 patients with haematological malignancy. Br J Haematol 2002;117:40-6.

14 Viscoli C, Girmenia C, Marinus A, et al. Candidemia in cancer patients: a prospective, multicenter surveillance study by the Invasive Fungal Infection Group (IFIG) of the European Organization for Research and Treatment of Cancer (EORTC). Clin Infect Dis 1999;28:1071-9.

15 Dellit TH, Owens RC, McGowan JE Jr, et al. Infectious Diseases Society of America and the Society for Healthcare Epidemiology of America guidelines for developing an institutional program to enhance antimicrobial stewardship. Clin Infect Dis 2007;44:159-77. 
16 Rosenberg DJ. Infections, bacterial resistance, and antimicrobial stewardship: the emerging role of hospitalists. J Hosp Med 2012;7(Suppl 1):S34-43.

17 Wenzel RP, Edmond MB. The impact of hospital-acquired bloodstream infections. Emerg Infect Dis 2001;7:174-7.

18 De Rosa FG, D'Avolio A, Corcione $S$, et al. Anidulafungin for Candida glabrata infective endocarditis. Antimicrob Agents Chemother 2012;56:4552-3.

19 Pappas PG, Kauffman CA, Andes D, et al. Clinical practice guidelines for the management of candidiasis: 2009 update by the Infectious Diseases Society of America. Clin Infect Dis 2009;48:503-35.

20 ema.europa.eu. http://www.ema.europa.eu/docs/it_IT/document_library/EPAR_ Product_Information/human/000788/WC500020673.pdf. Secondary http://www. ema.europa.eu/docs/it_IT/document_library/EPAR_Product_Information/human/ 000788/WC500020673.pdf

21 Cornely OA, Bassetti M, Calandra T, et al. ESCMID* guideline for the diagnosis and management of Candida diseases 2012: non-neutropenic adult patients. Clin Microbiol Infect 2012;18(Suppl 7):19-37.

22 De Rosa FG, Garazzino S, Pasero D, et al. Invasive candidiasis and candidemia: new guidelines. Minerva Anestesiol 2009;75:453-8.
23 Leon C, Ruiz-Santana S, Saavedra P, et al. A bedside scoring system ("Candida score") for early antifungal treatment in nonneutropenic critically ill patients with Candida colonization. Crit Care Med 2006;34:730-7.

24 Ostrosky-Zeichner L, Sable C, Sobel J, et al. Multicenter retrospective development and validation of a clinical prediction rule for nosocomial invasive candidiasis in the intensive care setting. Eur J Clin Microbiol Infect Dis 2007;26:271-6.

25 Paphitou NI, Ostrosky-Zeichner L, Rex JH. Rules for identifying patients at increased risk for candidal infections in the surgical intensive care unit: approach to developing practical criteria for systematic use in antifungal prophylaxis trials. Med Mycol 2005;43:235-43.

26 De Rosa FG, Manzione NA, Ranghino A, et al. Anidulafungin treatment in a kidney transplant recipient with hepatic damage. Mycoses 2011;54(Suppl 4):12-15.

27 Neoh CF, Liew D, Slavin M, et al. Cost-effectiveness analysis of anidulafungin versus fluconazole for the treatment of invasive candidiasis. J Antimicrob Chemother 2011:66:1906-15.

28 Wilke M. Treatment and prophylaxis of invasive candidiasis with anidulafungin, caspofungin and micafungin and its impact on use and costs: review of the literature. Eur J Med Res 2011;16:180-6. 